\title{
Surfactant Aggregates Templated by Lateral Confinement
}

\author{
Manaswee Suttipong, ${ }^{\dagger}$ Brian P. Grady, ${ }^{+}$and Alberto Striolo* ${ }^{\dagger}$ \\ ${ }^{\dagger}$ Department of Chemical Engineering, University College London, Torrington Place, \\ London WC1E 7JE, United Kingdom \\ ${ }^{\ddagger}$ School of Chemical, Biological and Materials Engineering, University of Oklahoma, \\ Norman, Oklahoma 73069, United States
}

\section{Corresponding Author}

*Email: a.striolo@ucl.ac.uk

\section{SUPPORTING INFORMATION}


1. Self-assembled surfactant structures in fully hydrophobic trenches of width $L=$ $0.99 L_{s}, 1.91 L_{s}$, and $3.75 L_{s}$

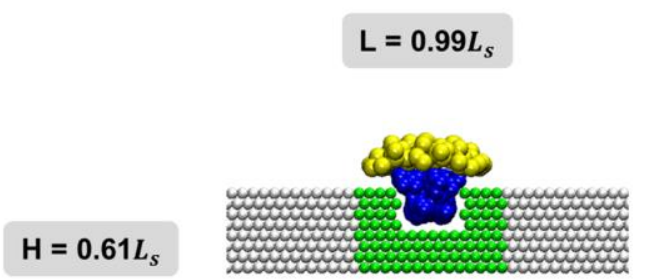

(a)

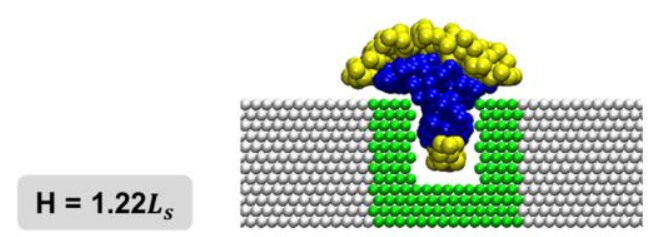

(b)

$\mathrm{H}=1.83 L_{s}$

$H=6.42 L_{S}$

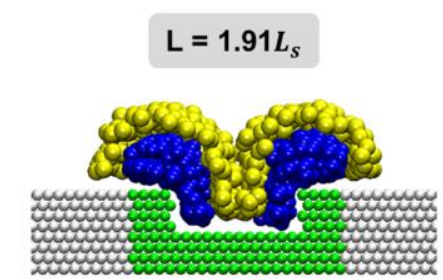

(e)

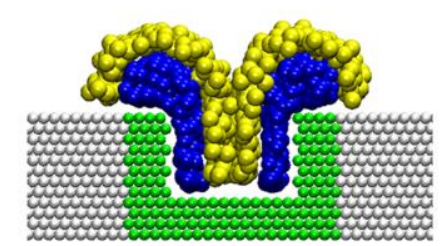

(f)

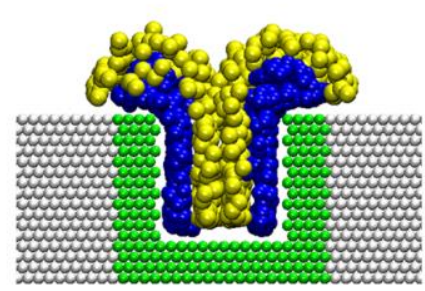

(g)

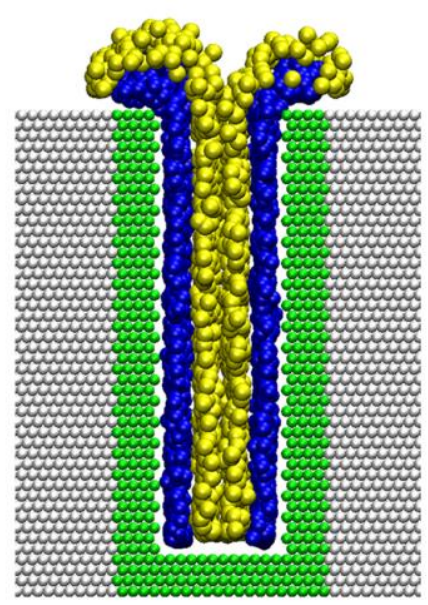

(h)

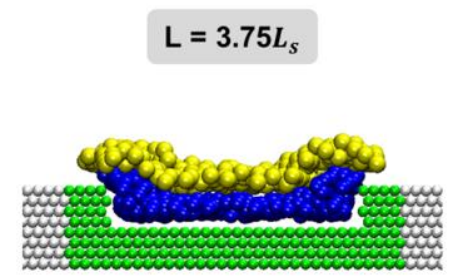

(i)

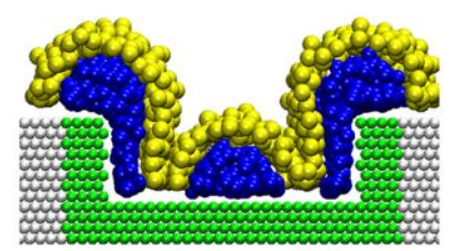

(j)

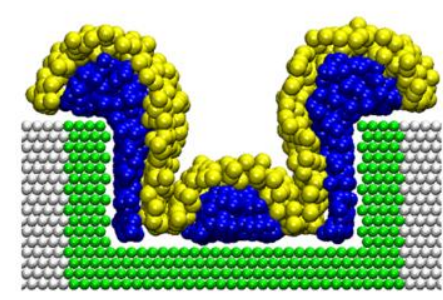

(k)

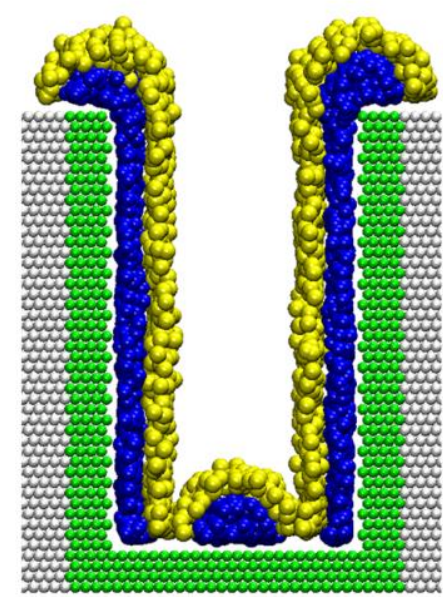

(l)

Figure S1. Equilibrium simulation snapshots of self-assembled surfactant aggregates within fully hydrophobic trenches of varying width $\mathrm{L}$ and depth $\mathrm{H}$. The results were obtained for trenches of width $\mathrm{L}=0.99 L_{s}, 1.91 L_{s}$, and $3.75 L_{s}$ (left to right panels) and depth $\mathrm{H}=0.61 L_{s}$, $1.22 L_{s}, 1.83 L_{s}$, and $6.42 L_{s}$ (top to bottom panels). The surfactant head groups, the surfactant tail groups, the hydrophobic surface sites, and the surfactant-repelling surface sites are shown as yellow, blue, green and grey, respectively. For clarity, water beads are not shown, nor are surfactants present in the bulk, i.e. not adsorbed on the trenches. $\mathrm{L}$ is expressed in units of one surfactant length $\left(L_{s}\right)$, which is $\sim 2.08 \mathrm{~nm}$ for sodium dodecyl sulfate. ${ }^{1}$ 
Equilibrium simulation snapshots obtained in fully hydrophobic trenches of width $\mathrm{L}=$ $0.99 L_{s}, 1.91 L_{s}$, and $3.75 L_{s}$ are shown in Figure S1. From top to bottom, the depth of the trench increases from $\mathrm{H}=0.61 L_{s}$ to $6.42 L_{s}$.

When $\mathrm{L}=0.99 L_{s}$, in the shallow trenches [Figure $\mathrm{S} 1(\mathrm{a}),(\mathrm{b})$, and (c)] the self-assembled structures (i.e., monolayer, bilayer, and multilayer) are similar to those reported in $\mathrm{L}=1.30 L_{S}$ (Figure 2 of the main text). As the trench depth increases further to $\mathrm{H}=6.42 L_{s}$ [Figure $\mathrm{S} 1$ (d)], unexpectedly, at the bottom of the trench no evidence of a monolayer is found. Instead, we observe hemi-spherical surfactant aggregates. In the region between the top and the bottom, our results suggest flat cylindrical micelles formed within the trench. Such aggregates can be seen clearly from the side view of simulation snapshot as shown in Figure S2. The tails are found to orient parallel to the trench walls to maximize tail-hydrophobic surface interactions, while the surfactant heads are found outside of the micelle, where water is present. The results suggest that lateral confinement has a stronger effect in the deeper trenches.

When $\mathrm{L}$ is twice the length of one surfactant molecule (i.e., $\mathrm{L}=1.91 L_{s}$ ), we do not observe confinement-induced structural transitions with changing the trench depth [Figure S1 (e) to (h)]. The surfactants yield a flat monolayer on both walls of the trench with the water film in between. At the top of the trench the surfactants agglomerate strongly on the trench edges.

In the wider trench (i.e., $\mathrm{L}=3.75 L_{s}$ ) it can be seen that surfactants are assembled in a flat monolayer when $\mathrm{H}=0.61 L_{S}$ [Figure $\mathrm{S} 1$ (i)]. As the trench depth increases further [Figure $\mathrm{S} 1$ (j), (k), and (l)], the surfactants yield a flat monolayer on both walls of the trench. This aggregate is similar to those obtained in Figure $\mathrm{S} 1$ (f), (g), and (h)]. At the bottom of the trench the surfactants compressed by these two monolayers form a hemi-cylindrical structure, with the tails at contact with the wall and the surfactant heads associated to water. 


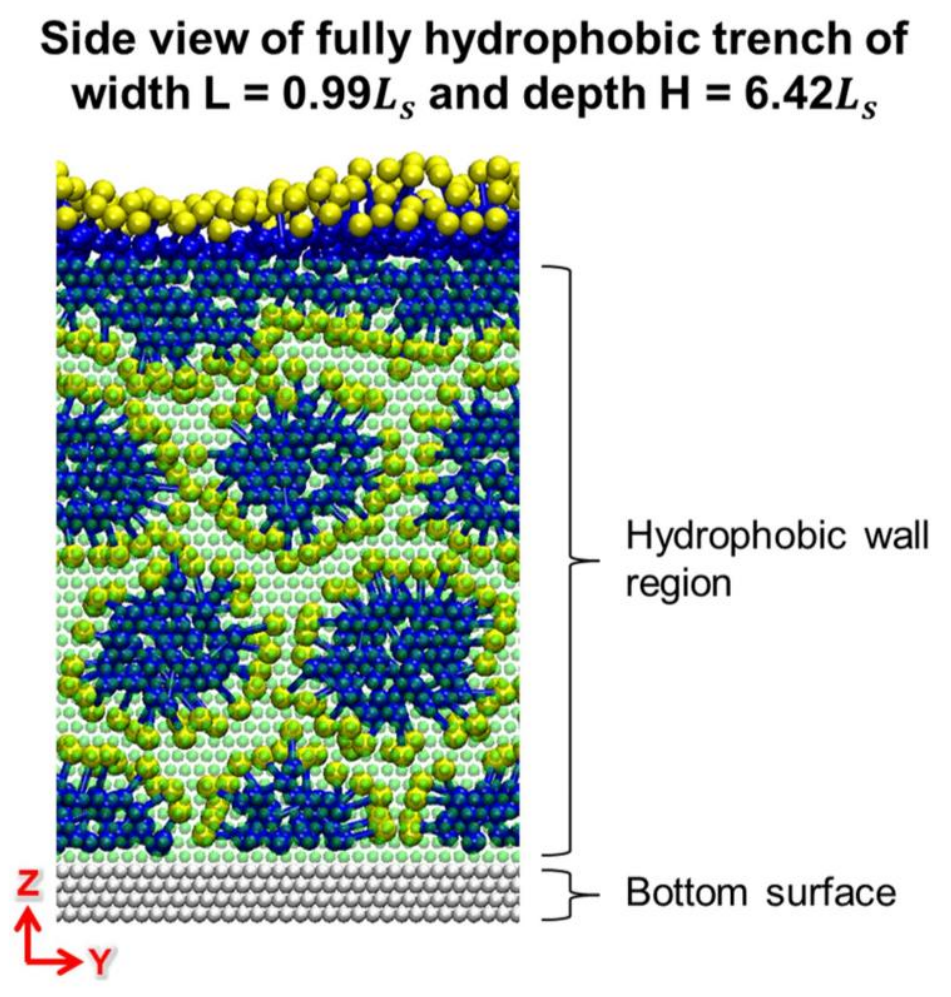

Figure S2. Side view of equilibrium simulation snapshots of self-assembled surfactant aggregates in fully hydrophobic trenches of width $\mathrm{L}=0.99 L_{s}$ and depth $\mathrm{H}=6.42 L_{s}$. The colour code is the same as that used in Figure S1. For clarity, water beads are not shown, nor are the surfactants present in the bulk.

\section{Water-trench system}

We performed simulations of water beads and trench in order to quantify the behaviour of water near the structured surface considered in our simulations. Trenches, both fully and partially hydrophobic, of width $\mathrm{L}=1.30 L_{s}$ with varying $\mathrm{H}=0.61 L_{s}, 3.06 L_{s}$, and $6.42 L_{s}$ were considered. Water shows similar behaviour within all trenches. Shown in Figure S3 is a representative simulation snapshot and density profile of water beads within the fully hydrophobic trench of $\mathrm{L}=1.30 L_{s}$ and $\mathrm{H}=3.06 L_{s}$. Note that $\mathrm{L}$ is $\sim 4$ times the diameter of one water bead. The results show that water wets the trench. Above the top of the trench $(\mathrm{Z}=$ $\left.\sim 3.6 L_{s}\right)$ water density reaches the constant value correspondent to the bulk density $(\sim 5$ beads per $r_{c}^{3}$ ). 

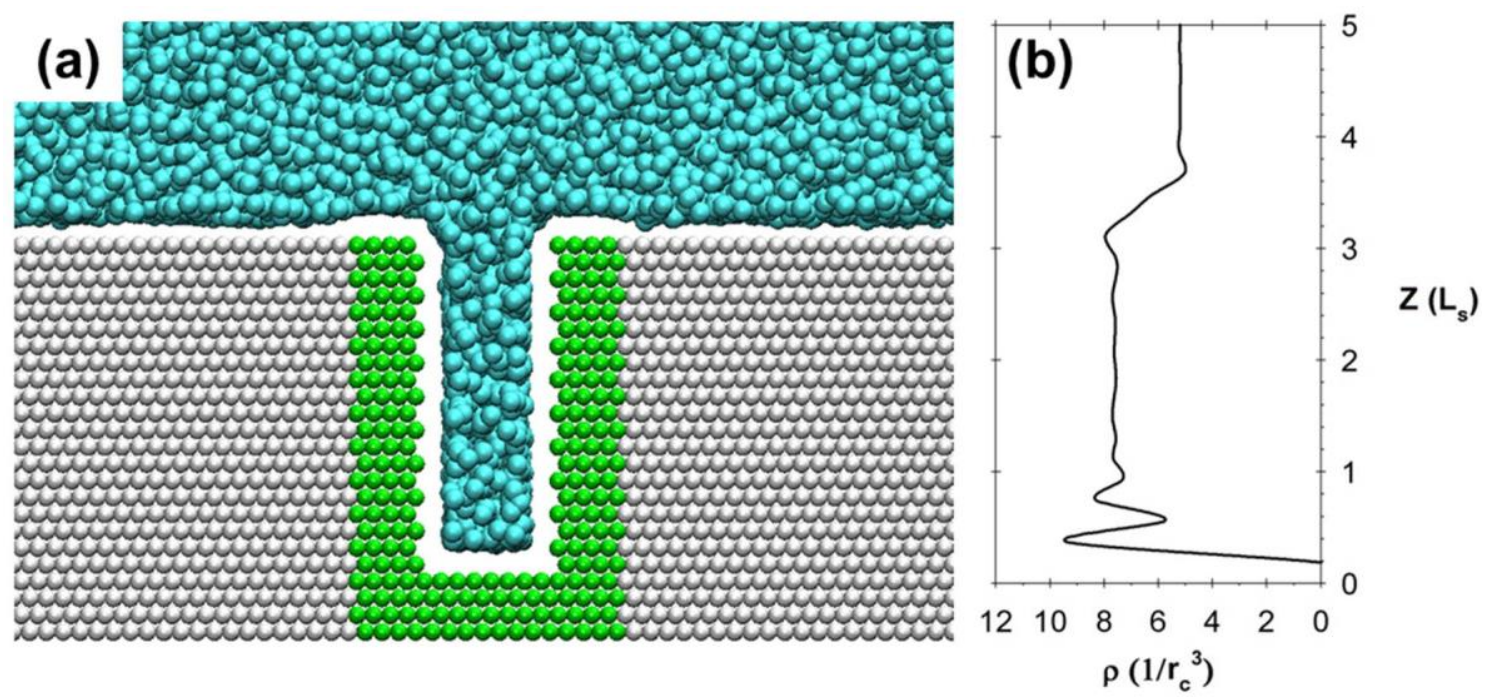

Figure S3. (a) Representative simulation snapshot and (b) water density distribution for the system of water and fully hydrophobic trench of width $\mathrm{L}=1.30 L_{s}$ and depth $\mathrm{H}=3.06 L_{s}$. In panel (a), water beads, hydrophobic surface sites, and surfactant-repelling surface sites are shown in cyan, green, and grey, respectively. In panel (b), density of water beads is expressed in number of beads per $r_{c}{ }^{3}$ and computed from the bottom trench surface along the $\mathrm{Z}$ direction. $r_{c}$ is defined as a cubic volume of radius (cut-off radius) and is of $9.0856 \AA$. $\mathrm{Z}$ is expressed in unit of one surfactant length $\left(L_{s}\right)$.

\section{REFERENCE}

(1) Zhao, H. T.; Nagy, K. L. Dodecyl Sulfate-Hydrotalcite Nanocomposites for Trapping Chlorinated Organic Pollutants in Water. J. Colloid Interface Sci. 2004, 274, 613-624. 